\title{
ON LOCAL SOLVABILITY OF LINEAR PARTIAL DIFFERENTIAL EQUATIONS NOT OF PRINCIPAL TYPE
}

\author{
BY PAUL R. WENSTON
}

Communicated by François Treves, July 23, 1974

I. Introduction. Necessary and sufficient conditions have been found [5], [6] , [7] for the local solvability of linear partial differential operators of principal type. An operator $P(x, D)$ of order $m$ on an open domain $\Omega \subset \mathbf{R}^{N}$ is said to be of principal type in $\Omega$ if $P_{m}(x, \xi)=0, x \in \Omega$, $\xi \in \mathbf{R}^{N} \sim\{0\}$ implies that $\nabla_{\xi} P_{m}(x, \xi) \neq 0$. L. Nirenberg and F. Treves [6], [7] have shown that if

(i) $P_{m}(x, D)$ has analytic coefficients,

(ii) for all complex numbers $z, \operatorname{Im}\left(z P_{m}\right)$ does not change signs in $\Omega$ along any null-bicharacteristic strip of $\operatorname{Re}\left(z P_{m}\right)$,

then $P(x, D)$ is locally solvable in $\Omega$. Hereafter we shall say that an operator $P(x, D)$ of principal type satisfies the N-T (Nirenberg-Treves) condition if $P_{m}(x, D)$ satisfies conditions (i) and (ii) above.

We remark here that for operators of principal type, local solvability depends only upon the leading terms. By contrast, for operators not of principal type one must consider lower order terms. Similar considerations arise in determining the hyperbolicity of operators with multiple real roots in their principal parts [1], [3], [4] .

With the above remarks in mind, we specify our problem. Let $P_{m}(x, D)=Q_{1}^{j_{1}}(x, D) \circ \cdots \circ Q_{k}^{j k}(x, D)$ with

(i) Each $Q_{i}(x, D)$ a homogeneous operator of principal type which satisfies the N-T condition.

(ii) $Q_{i}^{j_{i}}(x, D)=Q_{i}(x, D) \circ \cdots \circ Q_{i}(x, D) j_{i}$-times.

(iii) The $Q_{i}(x, \xi)$ 's having no common real roots except for $\xi=0$. We state the following definition.

Definition. An operator $T(x, D)$ of order $l<m$ is an admissible lower order perturbation of $P_{m}(x, D)$ if $\forall b \in C^{\infty}(\Omega), P_{m}+b T$ is locally solvable in $\Omega$.

AMS (MOS) subject classifications (1970). Primary 35A05; Secondary 35B20. 
In this note we state a sufficient condition for admissibility, and in the case where $\max \left\{j_{i}\right\}=2$, a weaker necessary condition. The proofs will be published elsewhere.

II. Sufficiency. Let $P=P_{m}+b T$, and define the operator $P_{j}(x, D)$ by $P_{j}(x, D)=Q_{1}^{\left[j_{1}-m+j\right]}(x, D) \circ \cdots \circ Q_{k}^{\left[j_{k}-m+j\right]}(x, D)$, where $[s]=$ $\max \{s, 0\}$. We assume that

$$
T=R_{l} \circ P_{l}+R_{l-1} \circ P_{l-1}+\cdots+R_{0} \circ P_{0}
$$

with order $\left(R_{j} \circ P_{j}\right) \leqslant j$. This immediately implies that

$$
P=P_{m}+R_{m-1} \circ P_{m-1}+\cdots+R_{0} \circ P_{0} .
$$

Many applications of the fact that for any two differential operators $L(x, D)$ and $H(x, D)$, the commutator $L(x, D) \circ H(x, D)-L(x, D) \circ H(x, D)$ is of order $\leqslant \operatorname{order}(L)+\operatorname{order}(H)-1$, enables us to state that if $\widetilde{P}_{j}(x, D)$ has precisely the same factors as $P_{j}(x, D)$, then there exist operators $\widetilde{R}_{j}(x, D)$ such that

$$
P=\widetilde{P}_{m}+\widetilde{R}_{m-1} \circ \widetilde{P}_{m-1}+\cdots+\widetilde{R}_{0} \circ \widetilde{P}_{0}
$$

We can then assume that $j_{1} \geqslant j_{2} \geqslant \cdots \geqslant j_{k}$, and we define the operators $S_{l}(x, D)$ by $S_{l}=Q_{1} \circ \cdots \circ Q_{j_{l}}, j_{l}=\max \left\{j_{i}: j_{i}-l \geqslant 0\right\}, l=1,2, \cdots, r=$ $\max \left\{j_{i}\right\}$. One notes that since the $Q_{i}(x, \xi)$ 's have no common real roots, $S_{l}(x, D)$ satisfies the N-T condition. Then since the formal adjoint $P^{\#}(x, D)$ has the same form as $P(x, D)$,

$$
P^{\#}=S_{1}^{\#} \circ \cdots \circ S_{r}^{\#}+R_{1} \circ S_{2}^{\#} \circ \cdots \circ S_{r}^{\#}+\cdots+R_{r}
$$

for appropriate differential operators $R_{j}(x, D)$. We now define the \|\|$\cdot \|_{p+s}$ norm by

$$
\|\varphi\|_{p+s}=\left\|\Lambda^{s}(D) \circ S_{2}^{\#}(x, D) \circ \cdots \circ S_{r}^{\#}(x, D) \varphi\right\|_{m_{1}-1} \quad \forall \varphi \in C_{0}^{\infty}(\Omega)
$$

where $m_{1}=\operatorname{order}\left(S_{1}^{\#}(x, D)\right)$. The following theorem is crucial.

THEOREM I. Let $x_{0} \in \Omega$. Then for all real numbers $s$, there exists $a$ neighborhood $\omega=\omega(s)$ of $x_{0}$ and a constant $C=C(s)$ such that

$$
\left\|P^{\#}(x, D) \varphi\right\|_{s} \geqslant C\|\varphi\|_{p+s} \quad \forall \varphi \in C_{0}^{\infty}(\omega) .
$$

Define $H_{p+s}(\omega)$ to be the completion of $C_{0}^{\infty}(\omega)$ under the \|\|$\cdot \|_{P+s}$ norm, and $H_{-P-s}(\omega)$ to be the completion of $C_{0}^{\infty}(\omega)$ under the norm 


$$
\|\varphi\|_{-p-s}=\sup _{\psi \in C_{0}^{\infty}(\omega)} \frac{\left|\langle\varphi, \psi\rangle_{L_{2}(\omega)}\right|}{\|\psi\|_{P+s}} .
$$

A standard argument then shows that $\forall f \in H_{-p-s}(\omega), \exists u \in H_{-s}\left(\mathbf{R}^{N}\right)$ such that $P(x, D) u=f$ in $\omega$.

III. Necessity. We have the following result on necessity when $\max \left\{j_{i}\right\}=2$.

THEOREM II. Let $P_{m-1}(x, D)$ be an admissible $m-1$ st order perturbation of $P_{m}(x, D)$. Then $\forall x_{0} \in \Omega, \xi^{0}$ a root of $Q_{1}^{j}\left(x_{0}, \xi\right) \cdots Q_{k}^{j k}\left(x_{0}, \xi\right)$ implies that $\xi^{0}$ is a root of the principal part, $\widetilde{P}_{m-1}\left(x_{0}, \xi\right)$, of $P_{m-1}\left(x_{0}, \xi\right)$.

We may assume that $x_{0}=0$. Standard arguments [2] then reduce the proof to showing that for arbitrary integers $K$ and $L, \exists b \in C^{\infty}(\Omega)$ such that

$$
\overline{\operatorname{Lim}_{\lambda \rightarrow \infty}} \sum_{|\beta|<L} \sup \left|D^{\beta t}\left(P_{m}+b P_{m-1}\right) v_{\lambda}\right|<\infty
$$

where

$$
v_{\lambda}=\lambda^{N+K+1} \sum_{\mu=0}^{r-1} \exp \left(i\left(\lambda^{2} u(x)+\lambda \psi(x)\right)\right) \varphi_{\mu} \lambda^{-\mu}
$$

with

(i) $\varphi_{\mu} \in C_{0}^{\infty}(\omega), u, \psi \in C^{\infty}(\omega), \omega$ an open neighborhood of 0 .

(ii) $\nabla u(0)=\xi^{0}, \operatorname{Im} u(x) \geqslant 0, x \in \omega$.

(iii) $\operatorname{Im} \psi(x) \geqslant a|x|^{2}, a>0, x \in \omega$.

$$
\left[{ }^{t}\left(P_{m}+b P_{m-1}\right) \exp \left(i\left(\lambda^{2} u(x)+\lambda \psi(x)\right)\right)\right] \exp \left(-i\left(\lambda^{2} u(x)+\lambda \psi(x)\right)\right)
$$

$$
\begin{aligned}
& =O\left(|x|^{2 r}\right) \lambda^{2(m-1)}+\text { lower order terms in } \lambda, \\
& \text { with } r=N+K+1+2(m-1)+2 L .
\end{aligned}
$$

\section{REFERENCES}

1. H. Flaschka and G. Strang, The correctness of the Cauchy problem, Advances in Math. 6 (1971), 347-379. MR 43 \#5147.

2. L. Hörmander, Linear partial differential operators, Die Grundlehren der math. Wissenschaften, Band 118, Academic Press, New York, 1963. MR 28 \#4221.

3. A. Lax, On Cauchy's problem for partial differential equations with multiple characteristics, Comm. Pure Appl. Math. 9 (1956), 135-169. MR 18, 397.

4. S. Mizohata and Y. Ohya, Sur la condition de E. E. Levi concermant des equations nyperboliques, Proc. Internat. Conf. on Functional Analysis and Related 
Topics (Tokyo, 1969), Univ. of Tokyo Press, Tokyo, 1970, pp. 177-185. MR 43 \#2349a.

5. L. Nirenberg and F. Treves, On local solvability of linear partial differential equations. I: Necessary conditions, Comm. Pure Appl. Math. 23 (1970), 1-38. MR 41 \#9064a.

6. - On local solvability of linear partial differential equations. II: Sufficient conditions, Comm. Pure Appl. Math. 23 (1970), 459-509. MR 41 \#9064b.

7. - A correction to: "On local solvability of linear partial differential equations. II: Sufficient conditions", Comm. Pure Appl. Math. 24 (1971), 279-288.

DEPARTMENT OF MATHEMATICS, CARNEGIE-MELLON UNIVERSITY, PITTSBURGH, PENNSYLVANIA 15213

Current address: Department of Mathematics, University of Georgia, Athens, Georgia 30602 